Relations industrielles

Industrial Relations

\title{
Organized Behavior: an Applied Psychological Approach, by Clay Hamner et Dennis W. Organ, Dallas, Texas, Business Publications Inc., 1978, 437 pp.
}

\section{Laurent Bélanger}

\section{Volume 34, numéro 1, 1979}

URI : https://id.erudit.org/iderudit/028949ar

DOI : https://doi.org/10.7202/028949ar

Aller au sommaire du numéro

Éditeur(s)

Département des relations industrielles de l'Université Laval

ISSN

0034-379X (imprimé)

1703-8138 (numérique)

Découvrir la revue

Citer ce compte rendu

Bélanger, L. (1979). Compte rendu de [Organized Behavior: an Applied Psychological Approach, by Clay Hamner et Dennis W. Organ, Dallas, Texas, Business Publications Inc., 1978, 437 pp.] Relations industrielles / Industrial Relations, 34(1), 204-205. https://doi.org/10.7202/028949ar

Tous droits réservés @ Département des relations industrielles de l'Université Laval, 1979
Ce document est protégé par la loi sur le droit d'auteur. L'utilisation des services d’Érudit (y compris la reproduction) est assujettie à sa politique d'utilisation que vous pouvez consulter en ligne.

https://apropos.erudit.org/fr/usagers/politique-dutilisation/ 
et de roulement. Lacomblez conclut que la flexibilité de la main-d'œuvre n'est pas aussi grande qu'on le croit généralement et que seule une approche socio-technique incluant «la prise en considération du monde extra-entreprise" (la région, son passé, son histoire) est susceptible de favoriser une adaptation réciproque de l'entreprise et de la main-d'œuvre régionale.

C'est la démarche que propose Eric VERBORGH dans une étude de caractère plus théorique intitulée «Organisation du travail et gestion socio-technique». Cet article décrit un cadre conceptuel et méthodologique connu, visant à considérer l'entreprise comme un système sociotechnique ouvert, en relation permanente avec son environnement. L'influence des facteurs externes sur le système social de l'entreprise y est particulièrement discutée ainsi que la primauté générale du système technique sur le système social. L'auteur offre un modèle explicatif des diverses influences s'exerçant sur et au sein de l'entreprise.

Enfin, deux articles plus brefs complètent cette sélection. Dans sa présentation intitulée "Relations entre services et intervention dans l'entreprise», Jean Pierre VANDENBOSH décrit quelques-unes des difficultés rencontrées par le psychologue lors de ses interventions auprès de l'entreprise. Pour sa part, Marcel BOLLE DE BAL discute brièvement des problèmes posés par l'apparition de méthodes perfectionnées de qualification du travail (job evaluation) et s'interroge sur l'avenir de celles-ci.

Bref, cet ouvrage présente à l'occasion des points de vue originaux sur des sujets connus ou déjà fouillés. Il ne s'agit pas cependant (les auteurs ne le prétendent pas non plus) d'un document de pointe ou d'un bilan des connaissances accumulées dans ce secteur.

Alain VINET

Université Laval

Organized Behavior: an Applied Psychological Approach, by Clay Hamner et Dennis W. Organ, Dallas, Texas, Business Publications Inc., 1978, 437 pp.
La majorité des programmes d'enseignement en administration, en psychologie, en éducation, en relations industrielles offrent sous une forme ou une autre des cours et des séminaires qui traitent du comportement des individus et des groupes au sein des organisations de travail. La diversité de ces cours et séminaires tant au plan du contenu que de la pédagogie utillisée soulève bien des questions quant à la nature même de ce champ de connaissances qu'est le comportement organisationnel. Existe-t-il, en effet, un noyau (core) de connaissances assez cohérent et assez homogène pour constituer une trame commune à tous ces enseignements? Les auteurs de cet ouvrage prétendent qu'à la lumière des développements récents de la psychologie et de la sociologie des organisations, il y a lieu de constater que le «comportement organisationnel» est beaucoup plus qu'un champ de connaissances et qu'il est en train d'accéder au statut de discipline académique avec un objet qui lui est propre, un mode de raisonnement et d'observation contrôlée adaptée des méthodes de recherches en sciences sociales. Leur prétention semble suffisamment fondée puisqu'ils ont réussi à identifier les thèmes qui constituent l'objet propre de ce domaine et qu'on retrouve de façon recurrente dans la diversité des enseignements. Les trois grands thèmes qu'ils ont retenus sont les suivants: le comportement individuel, le comportement des groupes et les effets de ces comportements sur l'efficacité et la «santé » d'une organisation de travail dans un contexte socio culturel donné. Chacun de ces grands thèmes sont traités selon un cheminement logique et historique du développement des connaissances, cheminement qui est susceptible de plaire à l'intelligence du lecteur. Les processus d'apprentissage et leur application en matière de formation et d'adrninistration de la discipline, les perceptions, les attitudes, la motivation, les différences individuelles, le stress, la satisfaction au travail, les programmes sociaux récents d'amélioration de la qualité de la vie au travail sont autant de sujets traités sur la rubrique «comportement individuel».

La structure et le fonctionnement du groupe de travail, les phénomènes des conflits, de pouvoir, de leadership et du contrôle constituent le deuxième grand thème 
de l'ouvrage. Un dernier chapitre, en guise de conclusion, aborde le sujet de l'influence des variables d'environnement sur le comportement des individus et des groupes. Il faut souligner ici que le traitement de certains sujets, tels que les processus d'apprentissage, les programmes de protection s'inspire largement de la théorie de Skinner qui fonde également le courant "B. Mod». (behavior modification, modification du comportement). Les auteurs ont pris soin d'en souligner les limites tout en faisant une utilisation prudente. Somme toute, cet ouvrage se distingue des autres (une dizaine) publiés récemment par l'effort de cohérence et par le langage utilisé qui demeure à la portée de tous ceux qui ne sont pas familiers avec ce domaine relativement nouveau de la connaissance.

\section{Laurent BÉLANGER}

Université Laval

La Contrattazione collettiva in Italia (19451977), a cura di Bruno Veneziani, Bari, Cacucci editore, 1978, 342 pp.

Democrazia politica e democrazia industriale, a cura di Silvana Sciarra, Bari, De Donato editore, 1978, $293 \mathrm{pp}$.

Ces deux ouvrages, respectivement sur la négociation collective en Italie de 1945 à 1977 et sur la démocratie politique et industrielle, ont en commun d'avoir été conçus et, pour une très bonne part, écrits par les collaborateurs de l'Institut de Droit du Travail de l'Université de Bari.

Gino Giugni, depuis plusieurs années professeur à l'Université de Rome, y fonda une véritable école juridique dans une perspective de relations industrielles, actuellement animée par ses anciens assistants et dignes successeurs, Bruno Veneziani et Gaetano Veneto, qui comptent parmi les contributeurs des deux livres.

Le premier volume occupe une place à part - et j'ajouterais: appréciable - dans l'effort d'évaluation, entrepris ces derniers temps, du système de négociation collective italien. Le Français que je suis ne peut pas ne pas remarquer au passage - et, en ce sens, l'appréciation de Veneziani, dans son exceliente préface, sur l'abondante littéra- ture spécialisée française, me semble trop généreuse - que nous sommes loin, de ce côté-ci des Alpes, à effectuer une démarche similaire, laquelle, en Amérique du Nord, bien sûr, apparaîtrait parfaitement naturelle.

Veneziani explique bien le procédé choisi: d'une part, saisir la physionomie spécifique du processus de négociation collective sur la base de branches d'industrie sélectionnées en raison de leur valence nationale et «méridionale» et, d'autre part, faire ressortir des traits typiques de ces diverses branches l'identité complexe du système italien de négociation, tel qu'il s'est développé depuis la fin de la deuxième guerre mondiale à nos jours.

Les branches choisies sont les suivantes: la métallurgie (Gaetano Veneto), la chimie, dans le secteur privé (Maurizio Ricci), le textile (Umberto Carabelli), les banques et les compagnies d'assurances (Tommaso Germano), le commerce (Domenico Garofalo), la construction - qui intéressait, visiblement, au plus haut point nos collègues nord-américains (Canio Lagala), et, last but not least, l'agriculture (Bruno Veneziani), dont l'oubli dans les études de relations industrielles, qui ne deviendraient pas pour autant agricoles ou pastorales, s'est trop fait ressentir jusqu'à présent dans maints pays du vieux continent.

Cette succession n'est, incontestablement, pas innocente, puisque les premières cités sont celles qui ont vu se manifester le syndicalisme le plus en pointe, celui qui a le plus contribué à façonner une configuration des relations du travail spécifiquement italienne, avec ses défauts et ses vertus, ses illusions et ses désillusions.

La négociation collective n'est pas absente, on s'en doutait, du second ouvrage ici signalé, et ce dès l'introduction de Silvana Sciarra sur la démocratie industrielle en Grande Bretagne, des Webb au rapport Bullock. Il est vrai, en effet, comme le remarque très bien l'auteur à propos du débat soulevé par ce rapport - qui «fonctionne dans le débat italien comme élément de provocation conceptuelle et non pas en tant qu'invitation à l'imitation" (Giugni) que l'acceptation d'une structure de participation aux organes ait suscité des inter- 